\title{
Application of the user-centred design process according ISO 9241-210 in air traffic control
}

\author{
Christina König ${ }^{\mathrm{a},{ }^{*}}$, Thomas Hofmann ${ }^{\mathrm{b}}$ and Ralph Bruder ${ }^{\mathrm{a}}$ \\ ${ }^{a}$ Institute of Ergonomics, Darmstadt University of Technology, Petersenstr. 30, 64287 Darmstadt, Germany \\ ${ }^{\mathrm{b}}$ Industrial Design, University of Applied Sciences Osnabrück, Vitihof 15a, 49074 Osnabrück, Germany
}

\begin{abstract}
Designing a usable human machine interface for air traffic control is challenging and should follow approved methods. The ISO 9241-210 standard promises high usability of products by integrating future users and following an iterative process. This contribution describes the proceeding and first results of the analysis and application of ISO 9241-210 to develop a planning tool for air traffic controllers.
\end{abstract}

Keywords: Air Traffic Control, User Centred Design Process, Participation, Interface Design, Iteration

\section{Introduction}

Designing future work systems in air traffic control is challenging, when requirements cannot be defined from the beginning and may change within the process [1]. Due to system complexity, any change of the air traffic controller's workplace can affect other, possibly distant, elements $[2,12]$ and should be conducted carefully.

Air traffic controllers have to maintain situation awareness in a setting of rapidly changing information, retrieved from a wide array of information sources. They have to decide with conflicting goals and high responsibility under time constraints. This temporarily causes a tremendous cognitive workload $[1,12,10]$.

Every human-machine-interface (HMI) in this context should support user's tasks as well as possible, and disturbances from non-functional interfaces are unacceptable. Design lapses in early development steps may lead to the failure of an entire project, when the product is not suitable for the concrete task or the future user will not be able or willing to use it. Mistakes of an air traffic controller might increase more fatal risks: If important information cannot be perceived and processed, a controller's situation awareness and decision making may be deficient. Accidents may occur. The same effects can be induced by inappropriate input concepts effecting erroneous user input. Both will increase controller's cognitive workload for these tasks and decrease their remaining capacity for other tasks.

Therefore HMI for air traffic controllers should be as usable [6] as possible. But how can this be achieved?

One main factor is the needs of the controllers: functions and information should be available at the right moment and presented in an adequate way to ensure they can and will be used. An early integration of future users in the design process could help $[7,3]$.

This research paper will describe the proceeding and first results of the analysis and application of ISO 9241-210 [7] in this specific context of use. It will exemplify how activities in a human-centred design can be performed and how the development and result of a product can be effected. This work is part of a $\mathrm{PhD}$ thesis in progress at TU Darmstadt regarding the implementation of ISO 9241-210 in air traffic control.

\footnotetext{
* Corresponding author. E-mail: koenig@iad.tu-darmstadt.de; Phone: +49 615116 6289; Fax: +49 6151162798
} 


\section{State of the art}

The development of human-machine-interfaces in air traffic control can follow various processes. Most of them try to prevent any delays and negative effects by using a concrete process model, although structure and documentation of those models differ widely.

The first type (waterfall model) uses concrete steps with specific activities. Every activity, e.g. design, will be performed only once in the whole process and by an expert for this domain. Every step has a concrete goal (milestone) which has to be completed before the next step can start. The user will usually be integrated in the last step, e.g. to evaluate the final outcome. Some modified waterfall models integrate prototyping to discuss and evaluate the status quo in the project team.

The second type uses a circular/iterative principle. Process stages will follow one another while every activity will be performed several times (e.g. design 1 , evaluation 1 , design 2 ). This iteration will be continued until the goal is achieved. User integration can be applied in the whole process or in some stages. Most of those models suggest multidisciplinary teams to ensure that every stage will be performed with the maximal expertise.

The classification of models in those two groups is usually difficult. Most models contain elements of both types, e.g. concrete steps with some iteration elements. Anyway, there seems to be a trend towards iterative models with the focus on user participation and multidisciplinarity.

The idea of future user involvement is used widely (e.g. $[3,8])$. Participation can be defined as contributing to something [13] and should increase user acceptance and commitment [8]. It will help the project team to understand users' needs and to define adequate requirements. Additionally some design problems can only be found in realistic settings when real users use a product in the same way they would in real life [7]. The more specific a product is, the more important the participation of users as experts for their tasks becomes.

\subsection{Intention of ISO $9241-210$}

A specific iterative design process model is presented in ISO 9241-210 [7]. This so-called "humancentred design" approach focuses on the needs of the future user in order to develop a usable humanmachine-interface. Principles are, among others, an explicit understanding and integration of users, their tasks and realistic environment, iterative processes and multidisciplinary skills and perspectives within the design teams.

After planning the design process, four activities are performed to complete one iterative circle.

The first activity focuses on the context of use, i.e. the characteristics of the users and relevant stakeholders, their tasks and the environmental conditions in which the system is used. Usually, an exhaustive comprehensive analysis is not possible immediately. So the context of use might be redefined or specified in future project phases.

The second activity consists in a specification of user requirements, inferred from the formerly defined context of use and relevant standards, ergonomic, and expert knowledge.

Design solutions will be produced in the third activity, trying to meet user requirements as well as possible. Depending on the project status, solutions may either be documented with simple sketches, detailed drawings, or even high elaborated functional or look-and-feel prototypes.

Finally the existing design solutions will be evaluated with user tests, expert evaluation, or both. If the requirements are fulfilled, the design process will be terminated. In the majority of cases some requirements will remain, or new requirements are added, so there is a need for iteration of some or all activities. The first concept will therefore be refined, completed, and optimized.

\subsection{Application in the Air Traffic Control Context}

The application of the human-centred design approach in air traffic control can be difficult for many reasons: tasks might be so specific and complex that the development team won't find a deep insight into all details. Even developing a simple display can affect the use of other systems and require the redesign of system components and work processes in distant segments of the system. Due to the complexity of the work system, optimizing one aspect might lead to an impairment of another.

Approved methods need modification to fit in this context of use: a great portion of an air traffic controller's work is mental and cannot be observed easily. User tests have to be conducted with only a few participants because a concrete interface is specific for one control tower and therefore the prospective user group is small. Every controller is highly specialized on his tower and has his own specific work strategies. Hence, user requirements will differ between towers 
and controllers and cannot be transferred easily. Providing a realistic context could mean integrating 30 people in a $3 \mathrm{D}$ real-time simulation just to assign the usability of a tool or, even worse, the improvement of one single aspect.

A HMI should be universal and flexible enough to suit to more than one tower, and specific enough to support the individual user.

Therefore, iterations and user participation are often avoided; although an iterative, human-centred proceeding might prevent some problems and result in a more effective, efficient, and satisfying product. So the question is to what extend ISO 9241-210 is suitable to develop a usable human-machineinterface for an air traffic controller. For this analysis this standard will be applied to a concrete project, passing mentioned activities and reflecting the utility and applicability of each activity and the whole iteration process.

\section{Case Study}

\subsection{Scope}

In the case study presented here, the centralization of three airports to one master controller working position raises many design questions. In order to supervise or control several airports, the controller needs an excellent planning, which can be supported by a planning tool. This paper will present some of the activities, focusing on the implications of design evaluation on requirements and design concepts.

It addresses a conceptual change, almost a paradigm shift, in work design of tower air traffic controllers. Today, they are situated directly at the airport that they are controlling. Large windows give a direct view on the runway and taxiway system and allow the perception of most information needed to control. In the future, today's towers might be replaced by virtual towers, which replace the out-ofthe-window view by a synthetic view, that is therefore independent of any spatial restrictions. Additionally, it would be possible to control more than one airport from one working position.

This project was part of the joint project iPort VICTOR, assigned by DFS Deutsche Flugsicherung $\mathrm{GmbH}$ (business unit tower) and supported by Bundesministerium für Wirtschaft und Technologie (BMWi).

\subsection{Aim of the interface}

The human-machine interface will become part of a tower controller's working position. As a planning tool, it will show the traffic of three regional airports, pointing out possible conflicts (i.e. situations when two aircrafts demand the action of the controller regardless which airport) and supporting the controller's mental model of the actual situation, the socalled "picture". This so-called master controller should be able to solve conflicts by rescheduling flights or delivering the control of one or two airports to a colleague (remote controller). This solution should be documented in the planning tool by a controller's input.

\subsection{Planning}

Before starting the human-centred design activities, the procedure and the goals have to be planned. Additional to normal project management activities, the integration of users had to be considered. As the future users are highly specialized and well trained to their tasks, the new working concept including new tools means a large shift and demands an immense imagination effort. So the project team decided to both integrate controllers' perspective and expert knowledge of an operation manager in addition to the multidisciplinary project team (design, ergonomics, computer science).

\subsection{1st loop}

At first the four activities of the human-centred design process were passed through completely.

\subsubsection{1st activity: Understand and specify the con- text of use}

Defining the context of use usually starts with the definition of the user. Here, the users will mainly be tower air traffic controllers, working at German regional airports and employed by The Tower Company, a subsidiary of DFS Deutsche Flugsicherung $\mathrm{GmbH}$ (German air traffic control). All of them are well-trained controllers and hold a license for at least one German regional airport, e.g. Dortmund or Leipzig-Altenburg. Active controllers can be male or female, between 20 and 55 years old. Their memory, concentration ability, mental stability, and stress resistance are usually above average [4]. Physical restrictions, e.g. visual impairments, are mainly excluded. Air traffic controller's work has high prestige, and the selection process is rigorous. Their decisionmaking can be considered as conservative, i.e. decisions are mostly based on facts, rules and standards. Safety is the highest priority. The planning tool will be part of their work equipment and be used only after an extensive training. 


\subsubsection{2nd activity: Specify the user requirements}

Air traffic controllers need to achieve and maintain situation awareness even with high information complexity, which increases cognitive workload [5]. Heintz [4] subsumes cognitive tasks of air traffic controllers on basic tasks, e.g. spatial orientation and selective attention. Situation surveillance and evaluation is partly based on the direct view out of the tower window [11]. Controller's interface should be appropriate for the concrete task and situation. If not, they will cause additional workload.

The requirements are based both on the demand of the controllers' tasks and ergonomic demands.

The first group of requirements specify main functions of the planning tool and were defined in former projects phases, e. $\mathrm{g}$.

[a1] Displaying the traffic of up to three airports.

[a2] Displaying (all) relevant information of an aircraft (e. g. callsign, route, slot, aircraft type).

[a3] Input by means of a computer mouse, keyboard or touch input device (TID).

[a4] Clear structured user interface.

[a5] Conflicts must be explicitly highlighted.

[a6] Conflict resolution through rescheduling flights or transferring the control of an airport to a colleague.

A second group of requirements ensure the ergonomic quality and usability of the planning tool. A main part of those requirements were adapted from ISO 9241-110 [7], e.g.

[e1] Suitability for the task: minimal resource demand (cognitive, visual), minimal attention needed for the interaction, fast and safe interaction, supporting the controller's tasks, giving a good overview at any time and at all three airports, apparent options for action (i.e. conflicts and possible solutions).

[e2] Error tolerance: prevent incorrect inputs or support their correction, intuitive and fail-safe interaction, consequences of inputs are transparent, indicated quickly (minimal latency) and clearly, consequences are at the user-expected position (usually nearby to the input)

[e3] Controllability: time pressure here mostly comes from the controller's task itself (processes like arriving cannot be halted) and is critical, the interaction with the interface must not create additional time pressure by limiting input time intervals, inputs should allow resumption after interruptions without any time limit, simple and fast interactions are preferred to minimize the attention needed to completely perform the interaction

[e4] Conformity with user expectations: interactions of master and remote controller are performed in the same way (drag \& drop to reschedule aircrafts), the interaction is close to common interaction principles.

Two criteria of this standard are considered less important for this planning tool:

Suitability for individualization: the user group is small, follows strictly defined standards and is trained in the same way, so the need for individualization is small, also the planning tool is a first demonstrator tool for the use in simulations, yet further development will show if there is need for individualization.

Suitability for learning: users will have an intensive training to use the final tool so learning will be performed before using the tool in real life

The final version of the planning tool will be measured with those criteria. To achieve best results, every design solution produced in the project has to be evaluated on whether criteria are fulfilled or not, and which changes are needed.

\subsubsection{3rd activity: Produce design solutions}

The 3rd activity consists in producing several design solutions to meet user requirements, and to implement prototypes.

The first concept is a schematic demonstration of possible elements and a possible display layout (figure 1). Five airports are represented with columns and different colours. A vertical time line represents the time dimension. This first sketch focuses on the general display layout. It allows understanding the flow logic and to identify inconsistencies and design challenges even in this early project stage. Both the design of aircraft representations and colours and sizes are less important. 


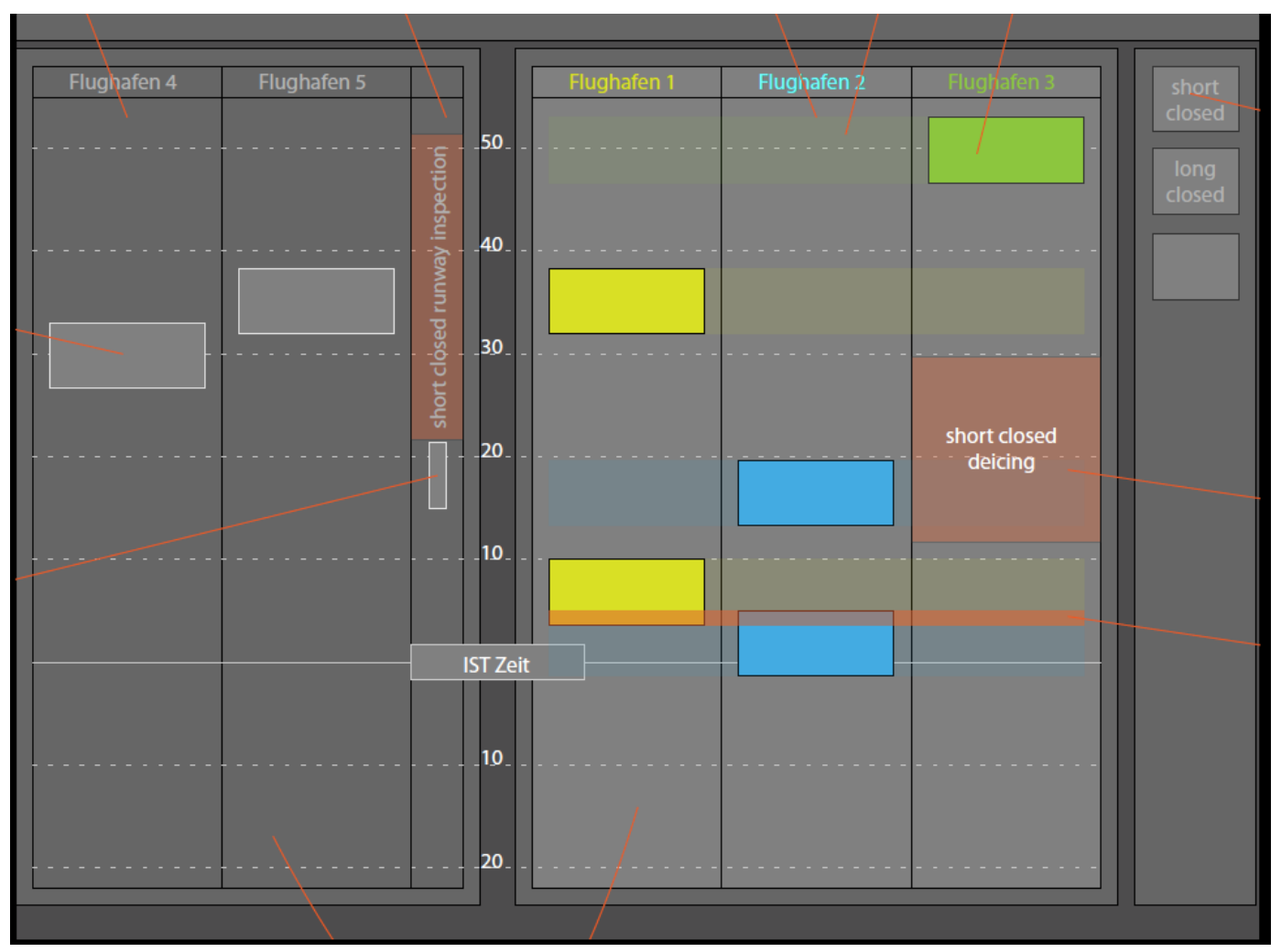

Fig. 1: First loop design

\subsubsection{4th activity: Evaluation}

The first concept already matches the requirements [a1] and [a4]. The other requirements remain to match in the next project phases. Additional requirements arise after the concept's evaluation with possible future users, e.g. choosing a monitor with at least 15 inches (requirement [a7]), and a continuous time progression [a8] instead of time steps. The suitability for the task [e1] could be estimated. The ergonomic requirements [e2] to [e4] cannot be applied in this project stage.

\subsection{2nd loop / 1st iteration}

As requirements still remain, iteration and reprocessing of at least some activities is necessary.

\subsubsection{2nd activity: Specify the user requirements}

The context of use doesn't necessitate any changes, except for the requirements. Some requirements are redefined (e.g. [a3], some are incorporated (e.g. [a7] and $[\mathrm{a} 8])$.

\subsubsection{3rd activity: Produce design solutions}

Based on the updated requirements the design concepts and first schematic sketches need to be refined and replaced with higher design quality. The focus is now on the design of the aircraft representations, so-called "label", and the colours. The current time is emphasized. Aircrafts under control of the master and the remote controller are distinguished by graphical means to ensure a fast and safe allocation (figure 2). 


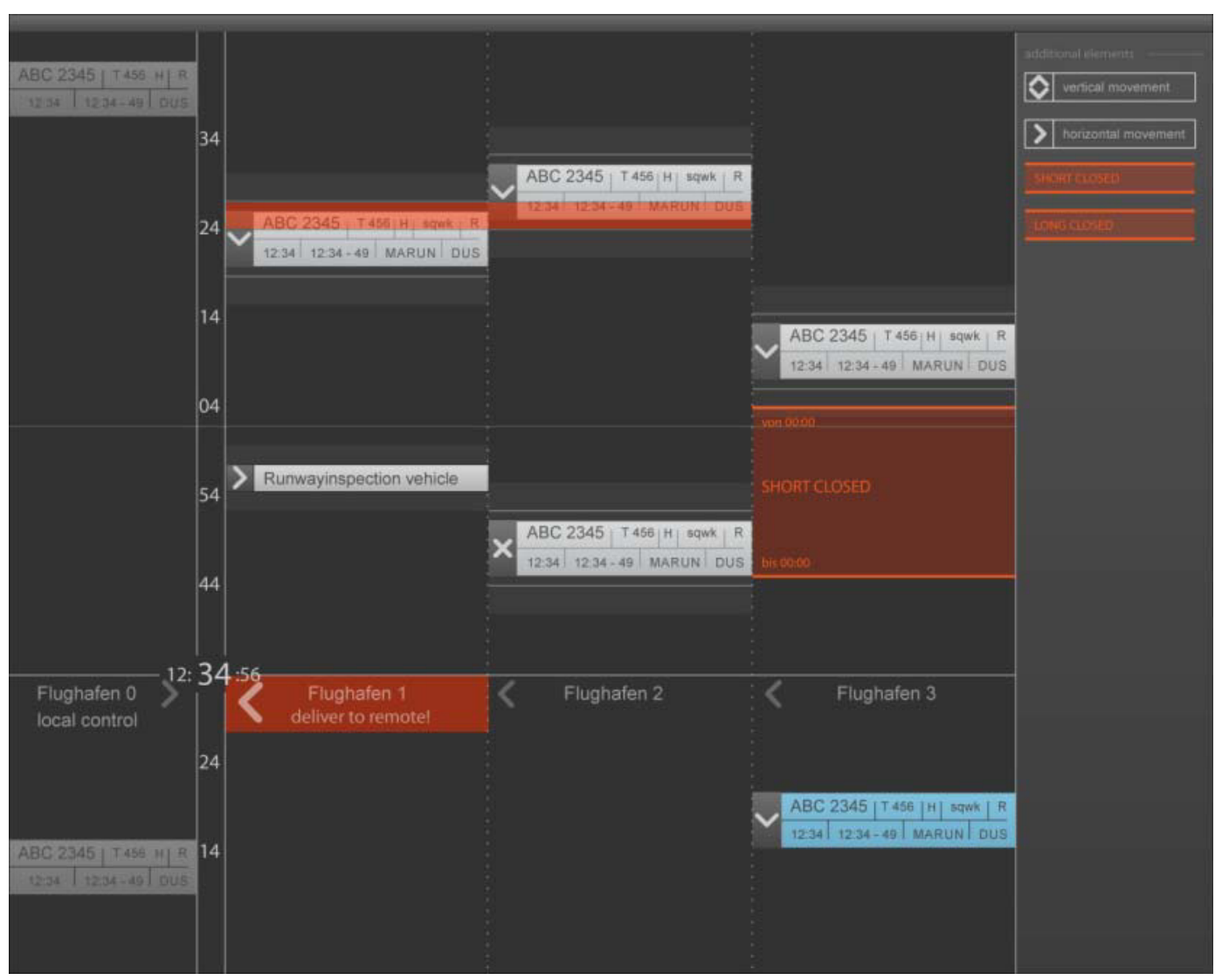

Fig. 2: Second loop design

The design closely follows existing, approved tools like the Tower-HMI or Tower-TID [1][9] to have higher conformity with user expectations. Most of those tools pick up the idea of paper strips and choose labels to represent aircrafts.

\subsubsection{4th activity: Evaluation}

This design takes into account that inputs will be done with fingers on a touch input device [a3]. The level of detail was reduced to point out the structure of the interface [a4] and to ensure a clear view. In particular requirement [a2] (displaying relevant information of an aircraft) is almost completely met.

The implementation in a software prototype showed deficits in error tolerance [e2]. Mapping aircrafts to a concrete landing or start-up time is difficult for users and should be supported by graphical means. The evaluation also finds deficits in the design and selection of this information. Conflicts are not highlighted sufficiently [a5] and are therefore difficult to solve [a6]. The suitability for the task is not sufficient either.
The conformity with user expectations [e4] can be assigned. Aircrafts can be rescheduled by moving the corresponding label with drag $\&$ drop, using a computer mouse or finger. Those inputs are widely used in other contexts and therefore well known in the user group. Still missing is an interaction to deliver an airport to a colleague.

\subsection{3rd loop / 2nd iteration}

Some requirements are met, but some still remain. So another iteration is required. 


\subsubsection{2nd activity: Specify the user requirements}

The context of use can be maintained. The requirements are readjusted according to the results of the evaluation in the 2 nd loop. One addition consists in a graphical differentiation between landing and starting aircrafts [a6]. Conflicts should be highlighted more clearly and explicitly (adjustment of [a5]). An aircraft label should cover the interval of the time line that it will need in reality [a8]. Additionally, the earliest and latest time of arrival or departure should be marked, i.e. master controller's the room of manoeuvre [a9]. Also an interaction to deliver airports to a remote controller or to take an airport over again is necessary (specification of [a6]).

\subsubsection{3rd activity: Produce design solutions}

The design has proceeded (figure 3 ). Conflicts are emphasized with red colour [a5], while other colours were reduced to bring out the dominance of conflict alerts. As red is almost the only colour in this concept, conflicts can be noticed at a short glance, while the overall design becomes more calm and clear [a4].

Grey lines above and beneath a label define the time an aircraft needs control and therefore keeps the attention of the master controller [a9]. The differentiation of arrivals and departures [a7] done with a symbol on the left part of the label.

Delivering and taking over of an airport [a6] can be documented with clicking on the name of the concrete airport.

\subsubsection{4th activity: Evaluation}

This design matches already most of the defined requirements. Three airports are represented [a1] with aircraft information needed for master control [a2]. The design is clearly structured and allows for a good overview [a4]. Conflicts are highlighted, and can be solved by rescheduling of aircrafts with drag $\&$ drop interactions [a3]. Inputs do not have any time limits.

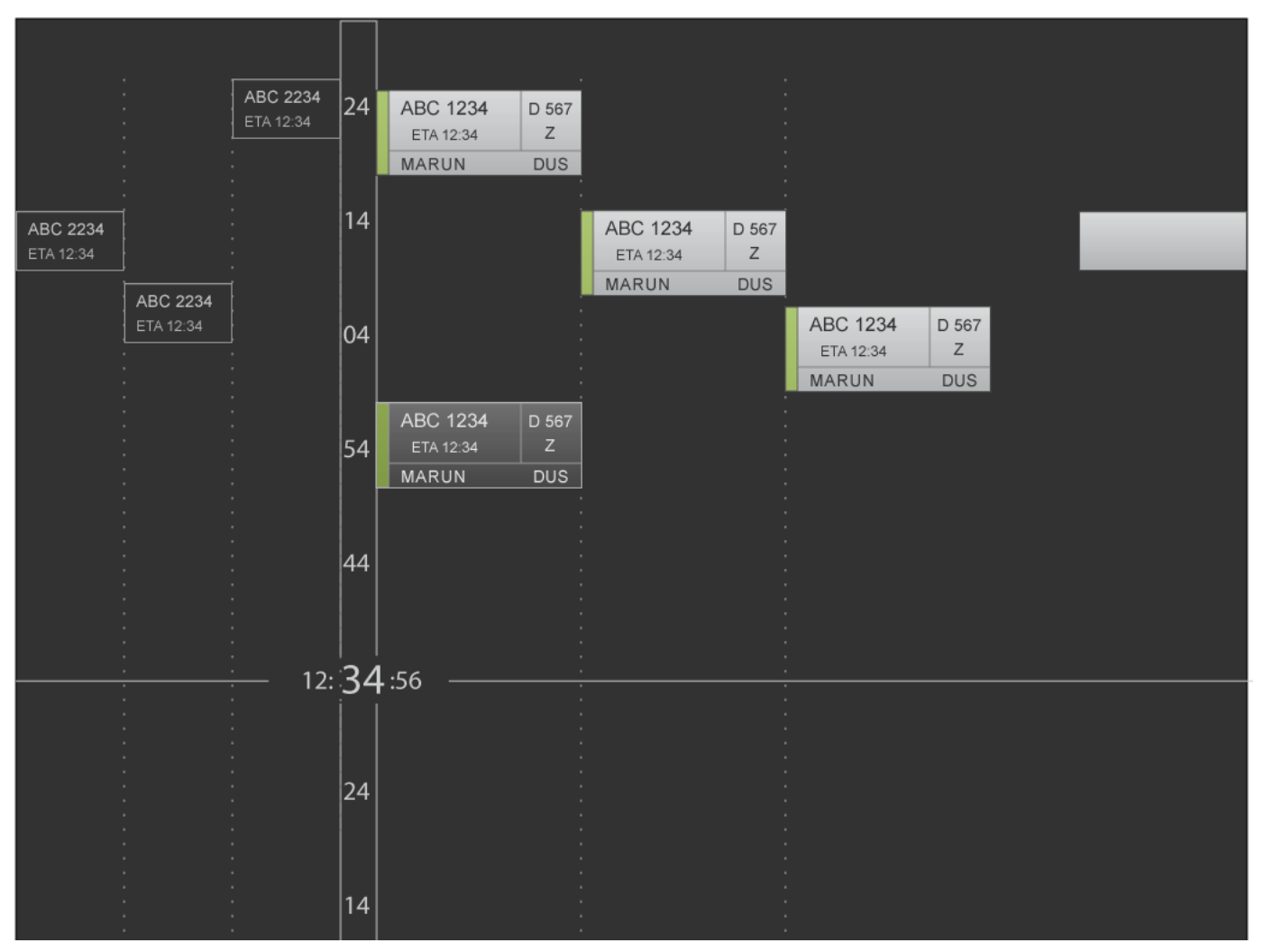

Fig. 3: Third loop design 
Using well-known interaction principles like clicking (to deliver and take over airports) and drag \& drop (to reschedule aircrafts) as well as the adaptation of common design elements like labels ensure a high conformity with user expectations [e4]. Arrivals and departures can be distinguished easily [a7]. The design solution shows the time demand of an aircraft [a8] as well as the limits for rescheduling [a9].

The suitability for the task [e1] and the error tolerance [e2] are validated in most instances. But a wellgrounded evaluation with intensive user tests under realistic working conditions is still necessary and will be performed in future project stages, e.g. with realtime simulations.

\section{Conclusion}

This analysis and application of ISO 9241-210 to the design of a specific interface gives first ideas about possible benefits and problems in the air traffic control context.

When applying user centred design process in air traffic control, some adjustments are recommended. Requirements and usability criteria should be defined with a fundamental knowledge about the controller's tasks. They cannot be defined in the first project stage completely, but need iterative adaptation. Both requirements from the task and ergonomic and usability criteria should be considered. Future users should be integrated to assure the product will support their work and will be accepted.

Prototypes are necessary even in early project stages to assess usability and conformity with the requirements, and to give users and project members a deep insight into design and interaction concepts. They help finding design problems and inconsistencies and correcting them before implementation.

Though some difficulties exist when ISO 9241210 is applied in such a specific and explicit nonconsumer product context, following this process has benefits. Participation can increase the acceptance and the suitability for the controller's task. Multiple iterations improve the interface continuously. The interdisciplinary approach ensures that all system components and their complex interaction are taken into account. Altogether, this human-centred design approach seems to fit well to design interfaces for air traffic control.

The next project steps comprise real-time simulations with realistic work scenarios. In this context, the planning tool will be evaluated and refined.
Further scientific work will focus on those usability criteria for high specialized, safety oriented and complex interfaces, and how those criteria can be measured in an economic and reasonable way.

\section{Acknowledgements}

Thanks are due to our project team member Tom Kamender for programming all prototypes and supporting the interface design, and to all prior project teams for developing the basic concept of the planning tool.

\section{References}

[1] Bergner, J., König, C., Hofmann, T., \& Ebert, H. (2009). An Integrated Arrival and Departure Display for the Tower Controller. Proceedings of 9th AIAA Aviation Technology, Integration, and Operations Conference, Hilton Head, SC, USA.

[2] Dörner, D. (2003). Die Logik des Misslingens: strategisches Denken in komplexen Situationen. Reinbek: RowohltTaschenbuch-Verl.

[3] Hall, R. R. (2001). Prototyping for usability of new technology. International Journal of Human-Computer Studies, 55(4), 485-501.

[4] Heintz, A. (1998). Anforderungsanalysen in der Flugverkehrskontrolle: Ein Vergleich verschiedener Arbeitspositionen. Hamburg: Institut für Luft- und Raumfahrtmedizin.

[5] Hopkin, V. D. (1995). Human factors in air traffic control. London: Taylor \& Francis.

[6] ISO 9241-11 (1998). Ergonomic requirements for office work with visual display terminals (VDTs). Part 11: Guidance on Usability. Geneva: ISO.

[7] ISO 9241-210 (2008). Ergonomics of human-system interaction. Part 210: Human-centred design process for interactive systems. Geneva: ISO.

[8] Jackson, T. F. (1980). System User Acceptance Thru System User Participation. Proceedings of the Annual Symposium on Computer Application in Medical Care, 3, 1715-1721.

[9] König, C., Hofmann, T., Bergner, J., \& Bruder, R. (2009). Inkrementelle nutzergerechte Etablierung eines TowerlotsenHMI. In S. Kain, D. Struve \& H. Wandke (2009): Mensch \& Computer 2009: Grenzenlos frei!? München: Oldenbourg, pp. 63-72.

[10]Manning, C. \& Stein, E. (2005). Measuring Air Traffic Controller Performance in the 21st Century. In B. Kirwan, M. Rodgers \& D. Schäfer (Hrsg.): Human Factors Impacts in Air Traffic Management. Hampshire: Ashgate Publishing.

[11] Marti, P. (1998). Structured task analysis in complex domains. In J. Annett \& N. Stanton (Hrsg.): Task Analysis. London: Taylor \& Francis, S. 137-149.

[12] Smolensky, M. W. \& Stein, E. S. (1998). Human Factors in Air Traffic Control. San Diego, US: Academic Press.

[13] Vroom, V. \& Jago, A. G. (1988). The New Leadership: Managing Participation in Organizations. Englewood Cliffs, N.J.: Prentice Hall. 